\title{
Etude expérimentale d'un écosystème sableux
}

\author{
II. Evolution des populations de bactéries et de méiofaune
}

\author{
S. Chamroux, G. Boucher \& P. Bodin \\ Station Biologique de Roscoff; Roscoff, France
}

\begin{abstract}
Experimental study on a sand ecosystem. II. Evolution of bacteria and meiofauna populations. A multispecific closed-circuit sand ecosystem was fuelled by regular addition of soluble amino acids during 28 months $\left(205 \mathrm{~g} \mathrm{C} \mathrm{m}^{-2}\right.$ year $\left.^{-1}\right)$. Bacteria and nematode densities undergo a similar evolution, divided into three main periods: the first 6 to 7 months (numerical decrease), from 6 to 7 to 20 months (numerical stability), after 20 months (numerical increase). Copepod densities are stable during the first seven months, then they exhibit an exponential increase until 14 to 15 month and, thereafter, a decrease. The inter-specific changes were followed for copepods and nematodes. One copepod species progressively overwhelms the fifteen others recorded. During 15 months, the nematodes specific diversity remains important. Although the number of rare species decrease progressively, the relative abundancy of the dominant species remains stable. After 15 month, the population changes according to a pattern of species succession.
\end{abstract}

\section{INTRODUCTION}

McIntyre et al. (1970) ont montré l'intérêt des colonnes expérimentales de sable pour comprendre les transferts de la matière organique dans la chaîne alimentaire, en insistant sur le rôle des bactéries et de la méiofaune (Nématodes et Copépodes essentiellement).

Nous avons réalisé un système comparable (Boucher \& Chamroux, 1976) en cultivant un sable sublittoral dont la faune d'origine était connue, particulièrement les Nématodes (Boucher, 1975; 1976; sous presse). Désirant contrôler la qualité de la matière organique fournie afin de mieux maîtriser le développement bactérien, nous avons utilisé comme nourriture des acides aminés sous forme soluble - substrat choisi comme étant le mieux assimilé par les bactéries marines ainsi que l'ont montré McLeod (1965), Baumann et al. (1971) et Bianchi (1971). L'emploi d'un circuit fermé a permis d'éviter l'introduction, en cours d'expérience, d'espèces étrangères au peuplement d'origine mais aussi de faire un bilan de la matière organique consommée.

Dans le présent travail, nous avons analysé l'évolution quantitative des constituants biologiques les plus représentatifs (bactéries, Copépodes, Nématodes). Nous avons ensuite suivi l'évolution qualitative des peuplements de Copépodes et de Nématodes. 


\section{MATERIEL ET METHODES}

Le schéma détaillé de l'écosystème expérimental utilisé dans ce travail ainsi que les techniques d'étude des bactéries et de la méiofaune ont été précédemment décrits (Boucher \& Chamroux, 1976).

\section{RESULTATS}

\section{Evolution du peuplement bactérien}

Dans les écosystèmes naturels ou expérimentaux, les bactéries interviennent de deux façons: elles servent de nourriture à un grand nombre d'organismes (méiofaune) et elles sont capables de modifier profondément et souvent très rapidement les caractères physiques et chimiques de leur environnement. Il paraît donc essentiel, dans tout écosystème expérimental, de connaître et même de prévoir le plus précisément possible leurs variations pour comprendre l'évolution des niveaux trophiques supérieurs.

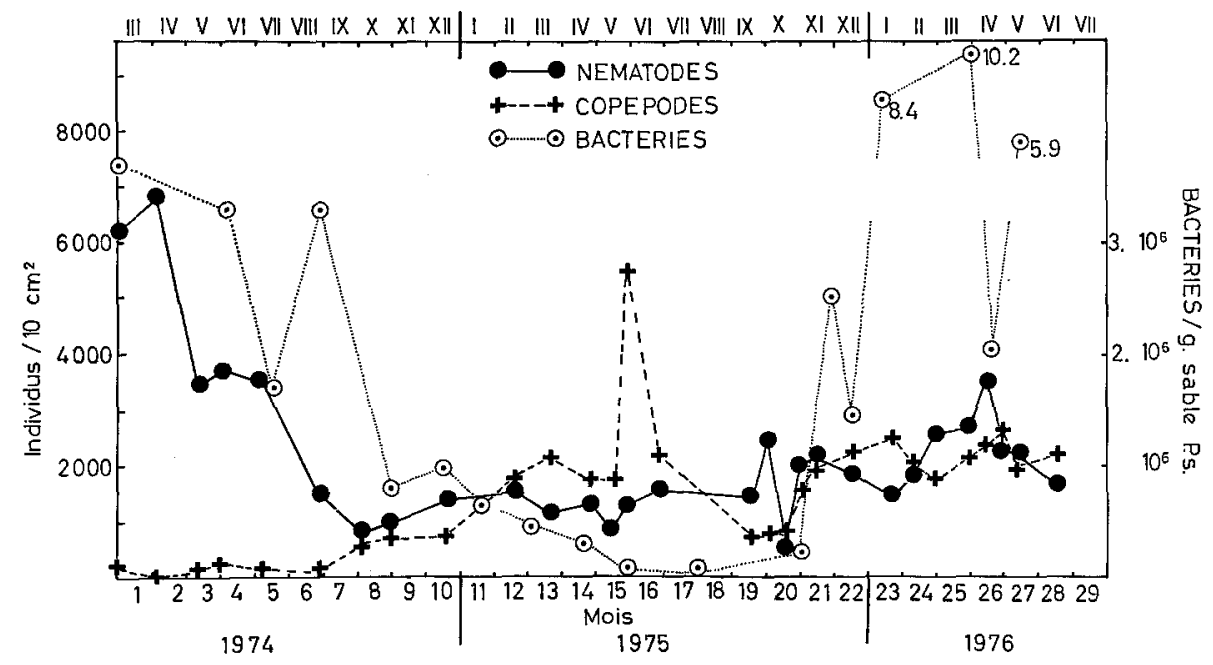

Fig. 1: Evolution des densités de Bactéries, Harpacticoïdes et Nématodes

La Figure 1 montre qu'après un temps de stabilisation relativement long, nous avons maîtrisé les fluctuations bactériennes pendant une période de 10 mois. Entre le 8 è et le 20è mois, elles se stabilisent à un niveau faible comparable à celui mesuré dans la nature. Pendant ce laps de temps, en maintenant leur croissance, par la quantité de nourriture fournie, à un taux de développement relativement constant et faible, nous avons favorisé des métabolismes de type oxydatif, les tensions d'oxygène mesurées à l'entrée et à la sortie du système étant toujours positives. Par certains points, le fonctionnement de l'écosystème, pendant cette période, pourrait s'identifier à un 
chémostat. En effet, l'apport en matière organique a été strictement contrôlé et réalisé sous forme d'un substrat défini et complètement assimilé au fur et à mesure de son addition (Boucher \& Chamroux, op. cit.). La quantité d'acides aminés fournie a servi d'élément nutritif limitant, régulant le taux de croissance bactérien.

Après 20 mois d'expérimentation, les populations bactériennes sont brusquement remontées à des taux relativement élevés. Nous avons observé, en même temps, une disparition complète de l'oxygène dans les parties profondes de la colonne de sédiment, mais nous n'avons pas cependant constaté d'empoisonnement du système.

Nous assistons donc à une évolution des populations bactériennes en trois phases. Les variations quantitatives, lors de ces trois périodes, sont d'amplitude suffisante pour pouvoir être rapprochées des variations concomitantes de la méiofaune.

\section{Evolution du peuplement de Copépodes \\ Etude quantitative}

\section{Evolution des densités}

La courbe des densités (exprimées en nombre d'individus $/ 10 \mathrm{~cm}^{2}$ ) est assez difficile à interpréter (Fig. 1). Cette courbe a été établie à partir de la moyenne des trois prélèvements de chaque échantillonnage.

Au cours du premier mois, la population voit son effectif diminuer, sans doute à la suite des perturbations causées par le remplissage du bac. (Ce problème est discuté plus loin à propos des Nématodes.) Cette diminution affecte peut-être plus particulièrement les formes mésopsammiques, car on n'en retrouve aucune dans le système expérimental.

Puis, durant une période de cinq mois, la courbe indique une lente progression de la population. Cette période correspond donc probablement au début du repeuplement de l'écosystème à partir d'un stock très appauvri.

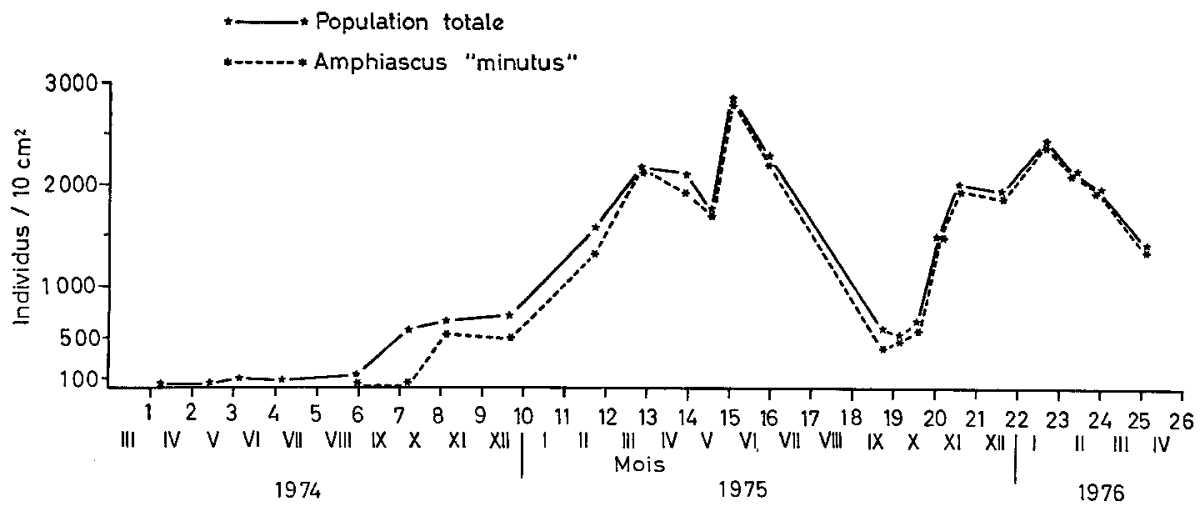

Fig. 2: Evolution comparée des densités d'Amphiascus "minutus" et de la population totale 
C'est alors qu'apparaissent les premiers individus de l'espèce Amphiascus "groupe minutus" (Claus) (cf. Lang, 1965; p. 255), espèce dont l'étude systématique est en cours. Dès le mois suivant (nov. 1974), Amphiascus "minutus" commence à prendre une grande importance dans la population des Copépodes Harpacticoïdes pour, finalement, la représenter presqu'entièrement à elle seule (Fig. 2). Il faut noter également que l'on peut voir de nombreux individus de cette espèce nager en pleine eau dans le bac.

On aboutit ainsi à un élevage à peu près monospécifique, dont la densité subit des fluctuations importantes.

Malgré les écarts assez nets de deux valeurs, on peut considérer que la portion de la courbe comprise entre le Gème et le 15ème mois indique une croissance exponentielle, avec un temps de doublement de la population de l'ordre de 75 jours. Cette période correspondrait à des conditions optimales de développement, en particulier pour Ampbiascus "minutus". Les densités atteintes au 15éme mois (en moyenne 5500 ind. $/ 10 \mathrm{~cm}^{2}$ ) sont environ 10 à 20 fois supérieures à celles observées dans le milieu naturel.

Aussitôt après, le nombre des Copépodes Harpacticoïdes diminue brutalement et, aux 19 ème et 20 ème mois, les densités passent par un minimum d'environ 700 à 750 ind. $/ 10 \mathrm{~cm}^{2}$.

Ensuite, les valeurs augmentent rapidement et oscillent finalement aux environs de 2000 ind. $/ 10 \mathrm{~cm}^{2}$, c'est-à-dire à un niveau comparable à celui des Nématodes, ce qui est assez rare dans la nature. Depuis le démarrage du système expérimental, on constate que les densités des Copépodes Harpacticoïdes varient souvent dans le sens inverse des densités de Nématodes (Fig. 1).

Il semble donc que l'on soit en présence d'une population qui se développe dans un milieu où la compétition interspécifique est très faible.

\section{Répartition verticale}

Un certain nombre de carottes ont été découpées en tranches de $1 \mathrm{~cm}$ d'épaisseur, et les animaux de la surface ont été isolés à l'aide d'une pipette. Les comptages correspondants nous ont permis d'avoir une idée de la répartition verticale des Copépodes Harpacticoïdes dans le substratum. Les résultats ont été calculés pour la période comprise entre le 8ème et le 16 ème mois.

A la surface du sédiment, on compte environ 8 à $45 \%$ de la population, composée en majorité de l'espèce Amphiascus "minutus". Le premier centimètre de sédiment renferme, selon les cas, de 30 à $82 \%$ de la population, ce qui, avec les individus de la surface, constitue de 72 à $94 \%$ de la population totale. En fait, la quasi-totalité de la faune harpacticoïdienne est installée dans les quatre premiers centimètres $d u$ sédiment de l'écosystème, alors que, dans le milieu naturel, il n'est pas rare de trouver des Harpacticoïdes jusqu'à $13 \mathrm{~cm}$ de profondeur. Ceci est sans doute à mettre en relation avec l'absence d'espèces typiquement mésopsammiques dans le système expérimental. 


\section{Etude qualitative}

Pour cette étude, un seul des trois prélèvements réalisés à chaque échantillonnage a été pris en considération.

Les 27 prélèvements inventoriés ont livré 16 espèces (Fig. 3), dont une (Proameira signata Por) est nouvelle pour la France, une autre (Harpacticus compsonyx Monard) est nouvelle pour l'Océan Atlantique, et une troisième (du genre Pseudolaophonte A. Scott) est nouvelle pour la science et sera décrite ultérieurement. Quelques espèces, en particulier du genre Stenbelia (Del.), n'ont pu être déterminées avec certitude.

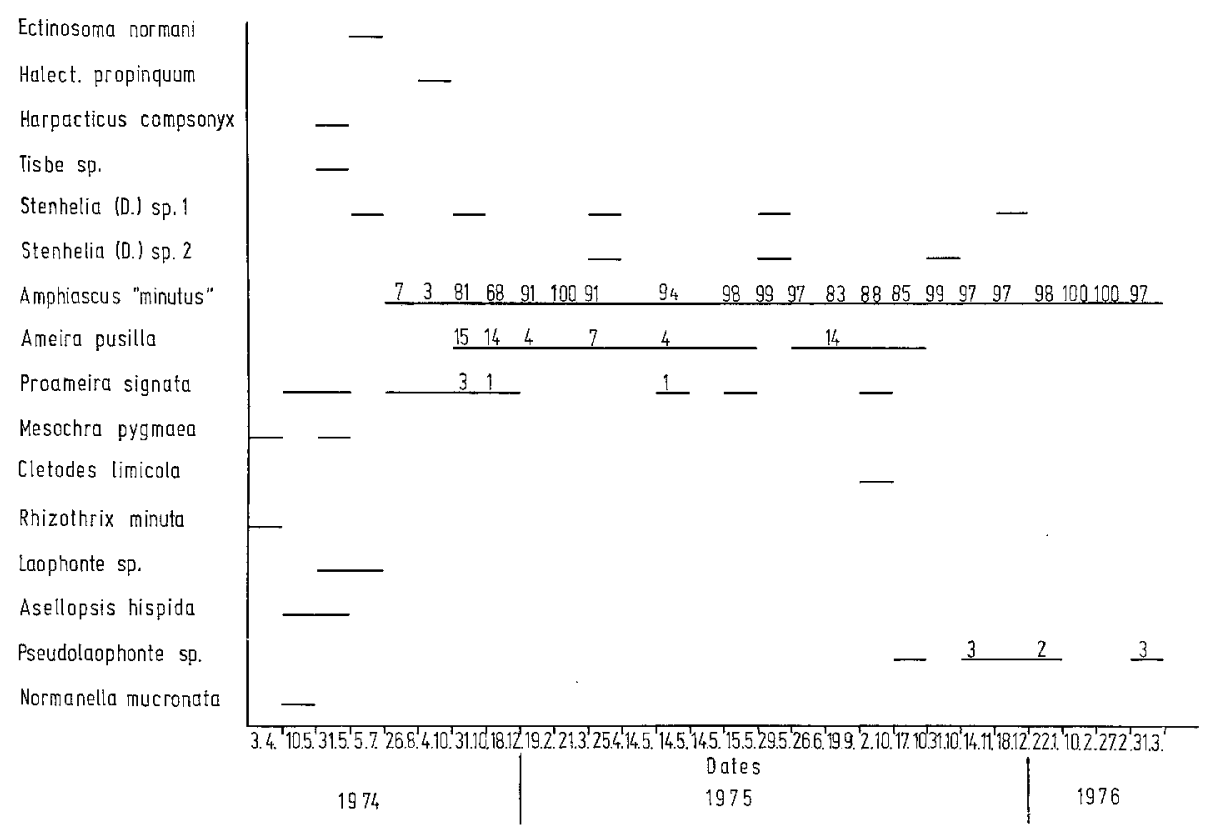

Fig. 3: Distribution des espèces de Copépodes Harpacticö̈des

En comparaison, deux prélèvements effectués dans le biotope d'origine, en Baie de Morlaix, ont été examinés et ont livré plus de 30 espèces, dont environ $50 \%$ de formes typiquement mésopsammiques. Pour les raisons exposées plus haut, aucune de ces dernières ne se retrouve dans l'écosystème, excepté Rbizotbrix minuta (T. Scott) que l'on peut à la rigueur considérer comme mésopsammique. Parmi les autres formes, 7 espèces ont été retrouvées dans le système expérimental.

On constate donc une diversité beaucoup plus faible dans l'écosystème que dans le biotope naturel. Sur l'ensemble des 27 prélèvements étudiés, on compte 71 signalisations d'espèces, ce qui correspond à une moyenne de 2,6 espèces par prélèvement, donc à une diversité extrêmement faible. Encore faut-il considérer que la quasitotalité de la population est représentée par trois espèces: Amphiascus "minutus", Ameira pusilla T. Scott et Proameira signata, avec une dominance écrasante de la première (Fig. 3). En effet, Amphiascus "minutus" représente presque toujours plus 
de $90 \%$ de la population, alors qu'elle semble rare dans le biotope d'origine. Elle n'apparaît dans nos listes qu'après 6 mois d'élevage mais, dès le 8ème mois, son importance est largement prépondérante. Les représentants du complexe "minutus" (Lang, 1965; p. 255) vivent habituellement dans les sables moyens à grossiers ainsi que dans les algues. Etant donné sa prolifération ici, on doit admette que l'environnement de l'écosystème lui convient parfaitement.

Ameira pusilla, rencontrée au 8ème mois, disparaît de nos listes un an après. C'est une espèce rare dont l'écologie est encore mal connue.

Rencontrée dès le second prélèvement, en mai 1974, Proameira signata est absente de plusieurs prélèvements et disparaît totalement après le 19ème mois (oct. 1975). D’après son auteur (Por, 1964), ses préférences écologiques vont aux fonds vaseux et sablo-vaseux.

Pseudolaophonte sp. est une espèce nouvelle (la troisième du genre, à notre connaissance) qui n'a été trouvée qu'à partir d'octobre 1975, soit près de 20 mois après la mise en eau de l'écosystème! Elle est assez régulièrement présente ensuite. Par contre, elle n'a pas été trouvée dans les deux prélèvements du biotope d'origine que nous avons inventoriés.

Des autres espèces, seule Stenhelia (Del.) sp. est assez régulièrement présente dans l'écosystème, du moins jusqu'en décembre 1975 (22ème mois). Les espèces de ce genre fréquentent en général les fonds vaseux ou sablo-vaseux.

La plupart des espèces vivant dans le système expérimental sont donc des formes essentiellement benthiques. Ceci est un point intéressant car, jusqu'à maintenant excepté les bons résultats obtenus avec Asellopsis intermedia (T. Scott) par Lasker et al. (1970), les élevages de longue durée ne concernaient le plus souvent que des espèces d'algues ou de pleine eau du genre Tisbe, Euterpina, Tigriopus, etc.

\section{Etude de l'espèce dominante}

Puisque Amphiascus "minutus" constitue la quasi-totalité des Harpacticoïdes depuis le 8ème mois, on peut tenter de se livrer à quelques calculs sur la population de cette espèce.

\section{Pourcentage de copépodites}

On trouve toujours des copépodites d'A. "minutus" (Fig. 4). Pour la période 1975-1976, il semble que les plus forts pourcentages se situent entre le 21ème et le 24ème mois ( 63 à $88 \%$ ). Les plus faibles valeurs se situent aux 12ème et 13ème mois (7 et $12 \%$ ), ainsi qu'à la fin du 20ème mois $(14 \%)$. On remarquera la belle homogénéité de l'écosystème au 15ème mois, lors des trois prises effectuées le 14 mai 1975 , à $10 \mathrm{~cm}$ l'une de l'autre: $38,7 \%-35,4 \%-36,0 \%$. Par contre, un prélèvement effectué dès le lendemain comporte un pourcentage nettement plus élevé de copépodites $(54,5 \%)$.

La reproduction de Ampbiascus "minutus" n'est donc pas continue dans l'écosystème, mais semble avoir lieu par phases successives. 


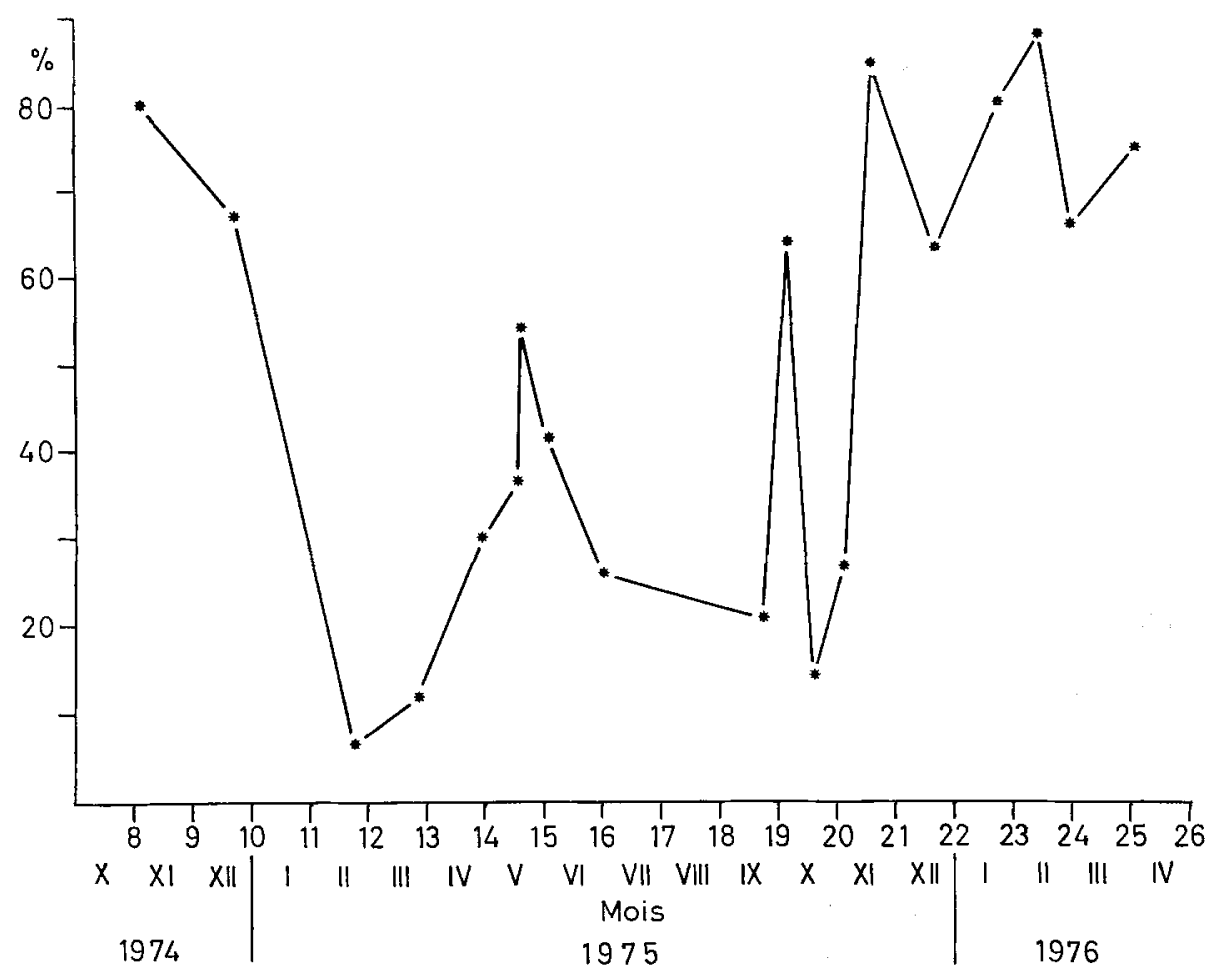

Fig. 4: Evolution du pourcentage de copépodites d'Amphiascus "minutus"

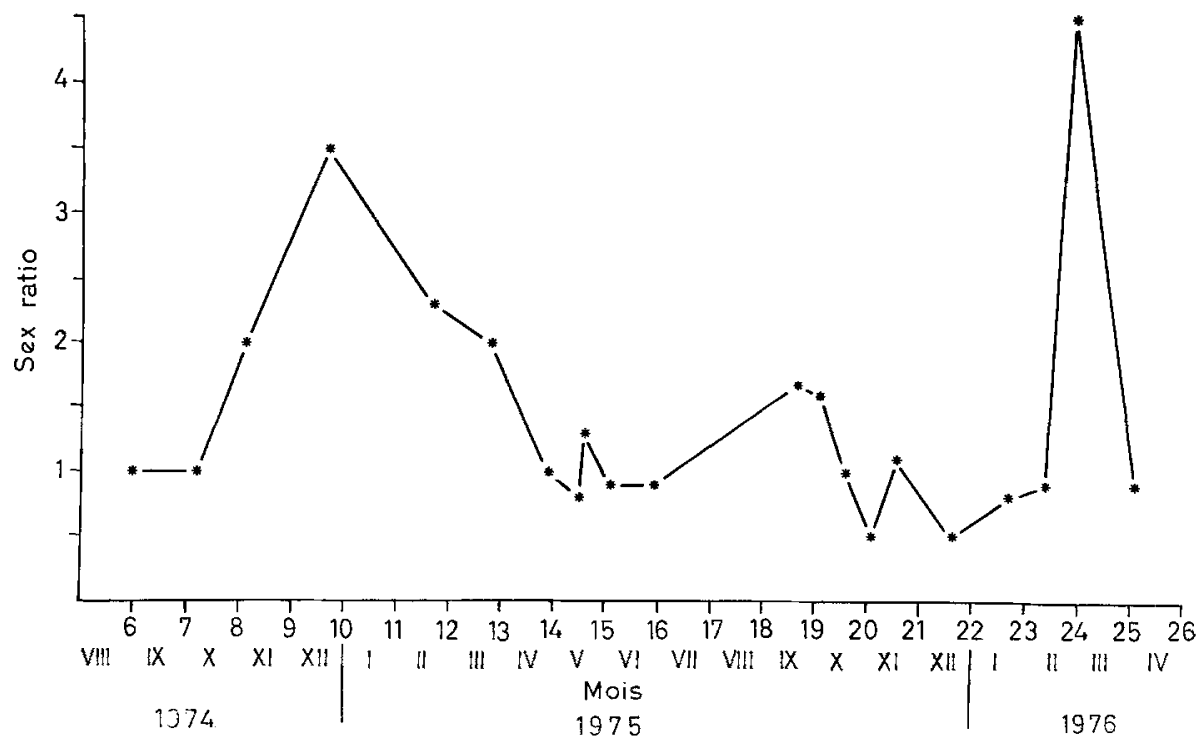

Fig. 5: Evolution du sex-ratio (mâles/femelles) chez Ampbiascus "minutus" 


\section{Sex-ratio}

Le graphique (Fig. 5) indique des variations assez importantes dans les proportions entre le nombre de mâles et le nombre de femelles. La moyenne générale $(1,4)$ est légèrement en faveur des mâles. Cependant, aux 21ème et 22ème mois, il y a deux fois plus de femelles que des mâles. Par contre, aux 10ème et 12ème mois, il y a environ trois fois plus de mâles que de femelles, et plus de quatre fois plus au 24ème mois.

\section{Répartition verticale}

Durant la période où les prélèvements ont été fractionnés en tranches de $1 \mathrm{~cm}$ d'épaisseur, on a pu remarquer (Tableau 1) qu'une proportion parfois importante des individus de l'espèce Ampbiascus "minutus" se trouvait à la surface du substrat. C'est le cas, notamment, aux 1 è̀me, 12 ème et 16 ème mois. Mais il arrive fréquemment aussi

Tableau 1

Répartition verticale d'Ampbiascus minutus (\%)

\begin{tabular}{|ccccccccc|}
\hline & \multicolumn{7}{c|}{ Mois } \\
Niveau & 9 & 10 & 11 & 12 & 13 & 14 & 15 & 16 \\
\hline 0 & 10 & 41,8 & 35,6 & 49,4 & 36,3 & - & 20,9 & 42,2 \\
1 & 81,7 & 50,9 & 56,9 & 26,5 & 54,7 \\
2 & 6,7 & 1,8 & 6,7 & 24,1 & 7,7 & 100 & 72,8 & 41,9 \\
$3-4$ & 1,7 & 3,6 & 0,8 & - & 1,2 & - & 4,2 & 7,8 \\
$5-6$ & - & 1,8 & - & - & - & - & 2,1 & 1,2 \\
\hline
\end{tabular}

que tous les individus soient enfouis dans le sédiment. Le premier centimètre renferme souvent plus de la moitié de l'effectif total de l'espèce, tandis que la quasi-totalité de cet effectif occupe les deux premiers centimètres du sédiment. Rares sont les représentants d'Ampbiascus "minutus" qui descendent au-dessous de $4 \mathrm{~cm}$ de profondeur.

\section{Evolutiondu peuplement de Nématodes}

\section{Evolution des densités}

Les densités de Nématodes très élevées au début de l'expérience (6190 à 6480 individus $/ 10 \mathrm{~cm}^{2}$ ) évoluent, en 7 mois, comme celles des bactéries, vers un niveau proche de celui observé dans le milieu naturel. Elles restent stables jusqu'au 19ème mois $(1159 \pm 174)$ avant de commencer à fluctuer assez brutalement en augmentant jusqu'à 3500 individus $/ 10 \mathrm{~cm}^{2}$ au 26 è mois.

L'existence d'une chute brutale du nombre de Nématodes pendant les 6 à 7 premiers mois de l'expérience souligne l'importance de la qualité du sédiment employé pour remplir un tel système expérimental. En effet, le sédiment d'origine récolté par 
Tableau 2

Comparaison des densités de Nématodes, Copépodes et Bactéries comptés dans des prélèvements simultanés obtenus par carottage in situ en plongée et par carottage sur du sable dragué (drague Charcot)

\begin{tabular}{|c|c|c|c|c|c|c|c|}
\hline \multicolumn{2}{|l|}{$\begin{array}{l}\text { Sable fin de la } \\
\text { Pierre Noire }\end{array}$} & \multicolumn{2}{|c|}{$\begin{array}{l}\text { Densités de } \\
\text { Nématodes } / 10 \mathrm{~cm}^{2}\end{array}$} & \multicolumn{2}{|c|}{$\begin{array}{l}\text { Densités de } \\
\text { Copépodes } / 10 \mathrm{~cm}^{2}\end{array}$} & \multicolumn{2}{|c|}{$\begin{array}{c}\text { Densités de } \\
\text { Bactéries/g PS }\end{array}$} \\
\hline $\begin{array}{l}\text { Carottage manuel } \\
\text { en plongée } \\
\text { in situ }\end{array}$ & $\begin{array}{l}1 \\
2 \\
3\end{array}$ & $\begin{array}{l}1215 \\
1715 \\
1611\end{array}$ & $\mathrm{~m}=1645$ & $\begin{array}{r}126 \\
95 \\
79\end{array}$ & $\mathrm{~m}=100$ & 0,6 à 0,8 & $10^{6}$ \\
\hline $\begin{array}{l}\text { Carottage sur } \\
\text { du sable dragué }\end{array}$ & $\begin{array}{l}1 \\
2 \\
3 \\
4 \\
5 \\
6\end{array}$ & $\begin{array}{l}5880 \\
5360 \\
6520 \\
6420 \\
6820 \\
6140\end{array}$ & $\mathrm{~m}=6190$ & $\begin{array}{l}260 \\
260 \\
200 \\
120 \\
280 \\
130\end{array}$ & $\mathrm{~m}=217$ & 1,1 à 3,8 & $10^{6}$ \\
\hline $\begin{array}{l}\text { Facteur de } \\
\text { concentration }\end{array}$ & & & 3,8 & & 2 & 1,8 à 4,7 & \\
\hline
\end{tabular}

dragage est nettement plus riche en Méiofaune que celui récolté parallèlement par carottage en plongée (Tableau 2). En ce qui concerne les bactéries, il est difficile de savoir si cet enrichissement est dû au mode de prélèvement comme pour la Méiofaune ou si le bouleversement de la structure du sédiment ne favorise pas un bloom bactérien dans le sable qui vient d'être dragué.

\section{Etude qualitative}

\section{Evolution des principales espèces du peuplement}

Plus de 130 espèces de Nématodes ont pu être identifiées dans les prélèvements. Du fait du nombre quelquefois limité de spécimens observés (152 individus par carotte en moyenne), seule l'évolution de treize espèces présentant à un moment donné une dominance supérieure à $5 \%$ a été retenue (Fig. 6). Parmi celles ci, il est possible de distinguer des espèces présentes au début de l'expérience et se développant pendant au moins 15 mois ( $n^{\circ} 1$ à 9 de la Figure 6), et des espèces rares ou absentes au départ ( $\mathrm{n}^{\circ} 10$ à 13) devenant largement dominantes en fin d'expérience.

Dans le premier groupe, apparaissent 3 types de développement: (1) Espèces largement dominantes dans le prélèvement d'origine devenant rares après le 7è mois: Leptolaimus tripapillatus, Microlaimus conspicuus. (2) Espèces abondantes pendant 15 mois devenant rares ou absentes ensuite: Chromaspirina renaudae, Actinonema celtica, Monbystera pusilla, Richtersia kreisi, Sabatieria celtica, Dichromadora cucullata. (3) Espèce abondante pendant toute la durée de l'expérience: Anticoma sp.

Nous observons donc un phénomène typique de succession d'espèces présentant trois phases. Les sept premiers mois correspondent à la raréfaction progressive de certaines espèces dominantes. La période du septième au quinzième mois correspond à la stabilité d'un groupe d'espèces caractéristiques du peuplement naturel. La période 


\section{Leptolaimus tripapillatus \\ 2 Microlaimus conspicuus 3 Chromaspirina renaudae 4 Anticoma sp. \\ 5 Actinonema celtica \\ 6 Mónhystera pusilla \\ 7 Richtersia kreisi \\ 8 Sabatiera celtica \\ 9 Dichromadora cucullata \\ 10 Paracanthonchus sp. \\ 11 Chromadoridae sp \\ 12 Monhystera sp. 2}

13 Neochromadora sp. 2

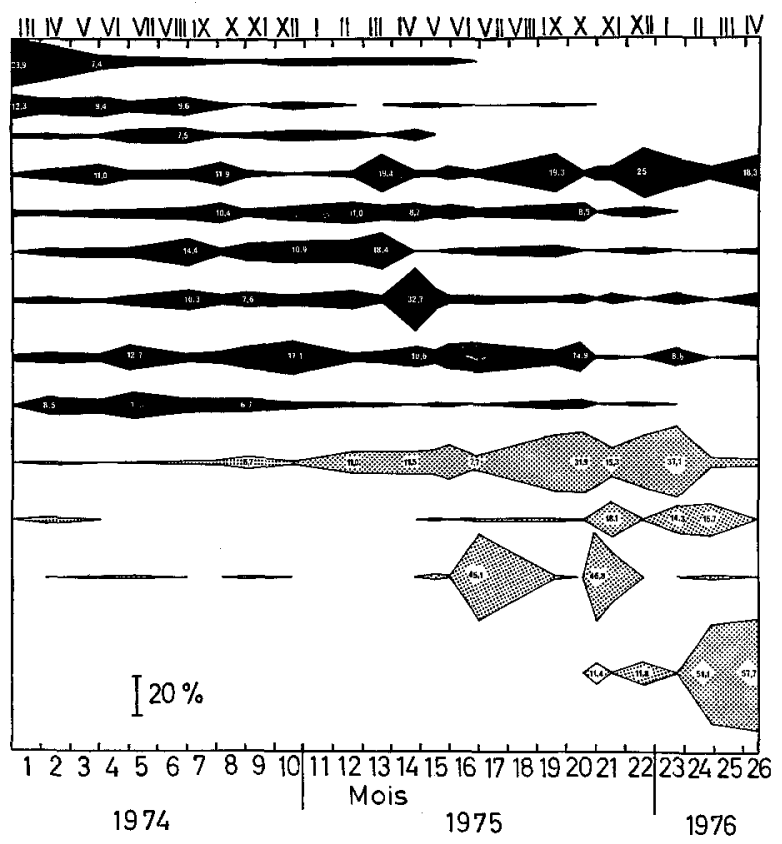

Fig. 6: Succession des principales espèces de Nématodes en fonction du temps

postérieure au quinzième mois est déterminée par l'apparition ou le développement explosif d'espèces rares au début de l'expérience et par le déclin plus ou moins rapide de la plupart des espèces caractéristiques.

Parmi les espèces dont la dominance ne dépasse jamais $5 \%$ mais qui sont caractéristiques du peuplement d'origine, certaines se développent pendant toute la durée de l'expérience. Il s'agit d'un groupe d'une trentaine d'espèces comprenant essentiellement des représentants de l'ordre des Desmodorida suceurs d'épistrates: Pselionema sp. et Cyttaronema sp., Epsilonema sp., Richtersia inaequalis, Desmodorella tenuispiculum, Ixonema sordidum, Neotonchus chamberlaini; de quelques représentants de l'ordre des Enoplida microphages tels Halalaimus sp., Oxystomina sp. ou carnivores tels que Enoploïdes sp.; de quelques Chromadorida carnivores: Choanolaimus sp., Latronema deconincki et d'un seul Monhysterida: Theristus bastiani.

\section{Evolution de la diversité du peuplement} lations:

Deux indices de diversité sont le plus souvent employés pour comparer des popu-

(a) l'indice de Fisher et al. (1943)

$S=\alpha \log e\left(1+\frac{\mathrm{N}}{\alpha}\right)$ où $S$ est le nombre d'espèces, $N$ est le nombre d'individus.

Cet indice surestime l'importance des espèces les plus rares mais corrige, dans 
les petits échantillons, la tendance des espèces à être collectées aussi rapidement que les individus.

(b) l'indice de Shannon-Wiener

$I_{\text {(bits) }}=-\sum_{\mathrm{i}=1}^{\mathrm{s}} \frac{n i}{N} \log _{2} \frac{n_{i}}{N}$

où $\frac{n_{i}}{N}=$ proportion de l'échantillon appartenant à la ième espèce

et $S=$ nombre d'espèces.

Cet indice tient peu compte des espèces rares. Il renseigne sur le mode de répartition des espèces dans le prélèvement. Toute variation de l'information fournie reflétera les variations de la distribution d'abondance des espèces.

Deux autres notions peuvent être aussi envisagées:

(c) l'indice de dominance utilisé par McNaughton (1968) qui est complémentaire de l'indice de diversité de Fisher et al. (1943).

$C=\frac{n 1+n 2}{N} \times 100$ où $n 1=$ abondance de la première espèce;

$n 2=$ abondance de la deuxième espèce.

(d) l'équitabilité de Pielou

$$
E=\frac{I \text { observé }}{I \text { maximum }} \times 100 \quad I \text { max. }=\log _{2} S \text { et définit un peuplement où toutes }
$$

les espèces ont une abondance égale.

Cet indice est complémentaire de celui de Shannon-Wiener car il renseigne sur la tendance à l'égalisation des effectifs.

Nous avons appliqué ces quatre indices complémentaires pour la détermination de la structure du peuplement (Fig. 7).

La diversité selon Fisher et al. (1943) décroît régulièrement pendant six mois, puis remonte brutalement à un niveau proche de sa valeur initiale avant de chuter de nouveau progressivement à un niveau plus bas que le précédent. Au quinzième mois, les oscillations de la diversité deviennent plus brutales et plus rapprochées. La raréfac tion du nombre d'individus par rapport au nombre d'espèces, bien que linéaire (baisse moyenne de la diversité de moitié en huit mois environ) dans l'ensemble, se fait par à-coups.

L'indice de dominance demeure assez constant (24\% en moyenne) pendant 12 mois avant de commencer à croître exponentiellement en oscillant de plus en plus fortement. Nous constatons que son augmentation est exponentielle alors que la chute de diversité est linéaire.

La diversité, calculée selon l'indice de Shannon-Wiener, reste très élevée pendant 12 mois $(4,20$ à 4,78). Elle varie peu et régulièrement dans le temps pendant 14 mois. Sa valeur oscille ensuite largement en décroissant rapidement (4,39 è 2,28 bits) jusqu'à la fin de l'expérience.

L'équitabilité augmente pendant sept mois puis elle décroît lentement jusqu'au 14 ème mois. Elle oscille ensuite rapidement en diminuant. La communauté est donc en équilibre pendant les 14 premiers mois puisque un changement lent de la diversité correspond à une communauté stable comme l'indiquent Hairston et al. (1968). 


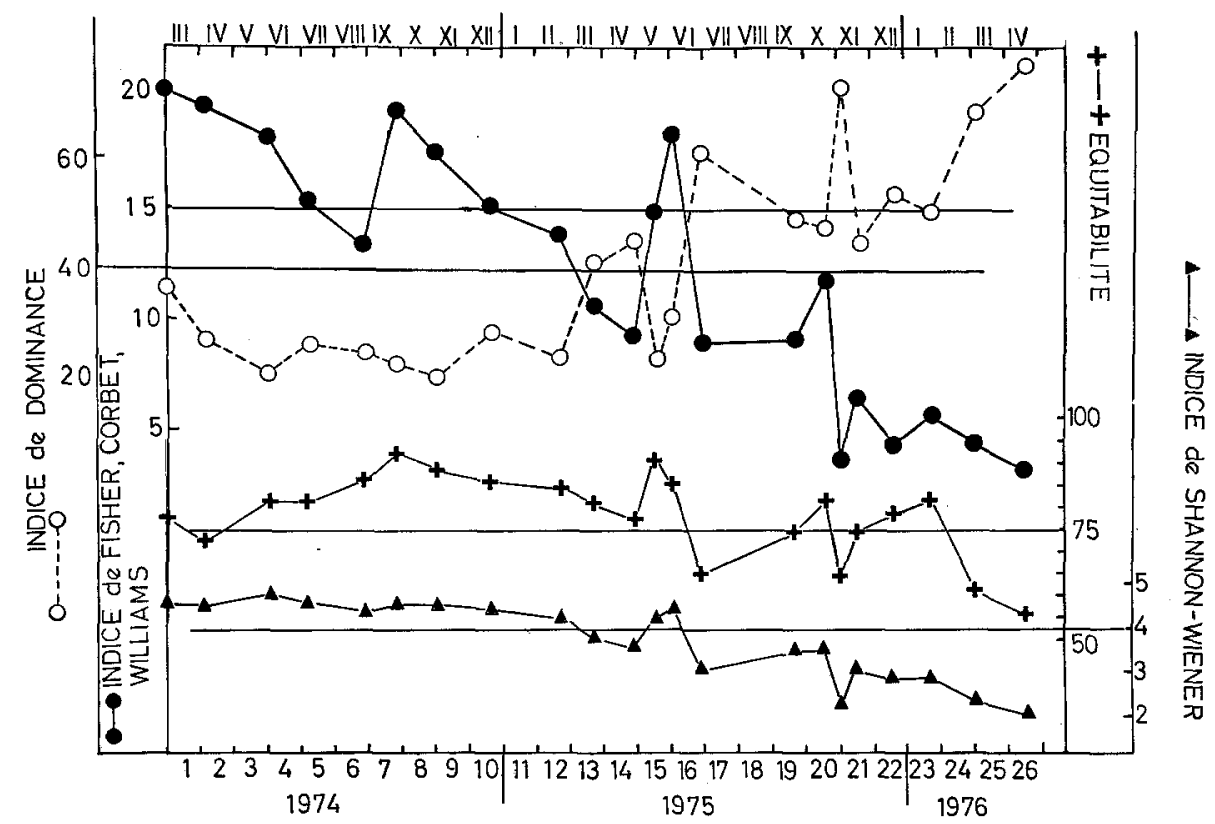

Fig. 7: Comparaison des indices de diversité et de dominance appliqués à l'étude du peuplement de Nématodes

L'emploi de l'indice de Shannon-Wiener semble mieux adapté que celui de Fisher et al. (1943) pour caractériser l'évolution du peuplement. Il tient essentiellement compte des espèces dominantes et élimine l'effet de mosaïque des nombreuses espèces rares habituellement recensées dans un faible volume de sédiment.

\section{Composition du peuplement d'origine}

Le Tableau 3 montre que la diversité trouvée dans le sable dragué est nettement plus faible que celle calculée dans les carottages en plongée mais aussi que son indice de dominance est beaucoup plus fort. Le mode de prélèvement par dragage, seul utilisable pour obtenir un grand volume de sédiment nécessaire au remplissage du bac,

Tableau 3

Comparaison des indices de diversité et de dominance dans les prélèvements récoltés en plongée et par dragage

\begin{tabular}{|ccccc|}
\hline Conditions & $\begin{array}{c}\text { Indice de } \\
\text { Fisher et al. }\end{array}$ & $\begin{array}{c}\text { Indice de } \\
\text { Shannon }\end{array}$ & Equitabilité & $\begin{array}{c}\text { Indice de } \\
\text { dominance }\end{array}$ \\
\hline plongée & 26,8 à 33,3 & 5,30 à 5,71 & 81,23 à 86,85 & $\begin{array}{c}13,8 \text { à } 18,09 \\
\text { m }=16,45 \\
36,1\end{array}$ \\
dragage & 20,3 & 4,53 & 78,37 &
\end{tabular}


Tableau 4

Changements des dominances de certaines espèces de Nématodes dus au mode de prélèvement du sédiment

\begin{tabular}{|llcc|}
\hline Régions & \multicolumn{1}{c}{ Espèces } & $\begin{array}{c}\text { Dominance dans les } \\
\text { carottes réalisées } \\
\text { en plongée }\end{array}$ & $\begin{array}{c}\text { Dominance dans les } \\
\text { carottes réalisées } \\
\text { sur sable dragué }\end{array}$ \\
\hline surface & $\begin{array}{l}\text { Rhynchonema ceramotos } \\
\text { Xyala riemanni }\end{array}$ & 3,52 & 0 \\
& profondeur & 1,93 & 0 \\
& Cylindrotheristus divertens & 2,44 & 0 \\
& Leptonemella granulosa & 0,94 & 0 \\
& Richtersia kreisi & 7,30 & 2,1 \\
couches & Leptolaimus tripapillatus & 0,7 & 23,8 \\
moyennes & Microlaimus conspicuus & 7,22 & 12,3 \\
\hline
\end{tabular}

transforme donc la structure du peuplement en même temps qu'il l'enrichit. La composition faunistique est changée (Tableau 4) par disparition totale au partielle de quelques espèces localisées en surface, mais surtout de la plupart des espèces de profondeur. Elle est aussi transformée par la concentration de certaines espèces réparties habituellement dans l'ensemble de la colonne de sédiment. Le peupiement utilisé pour remplir le bac est donc au départ déséquilibré et son évolution dans les conditions expérimentales en est affectée.

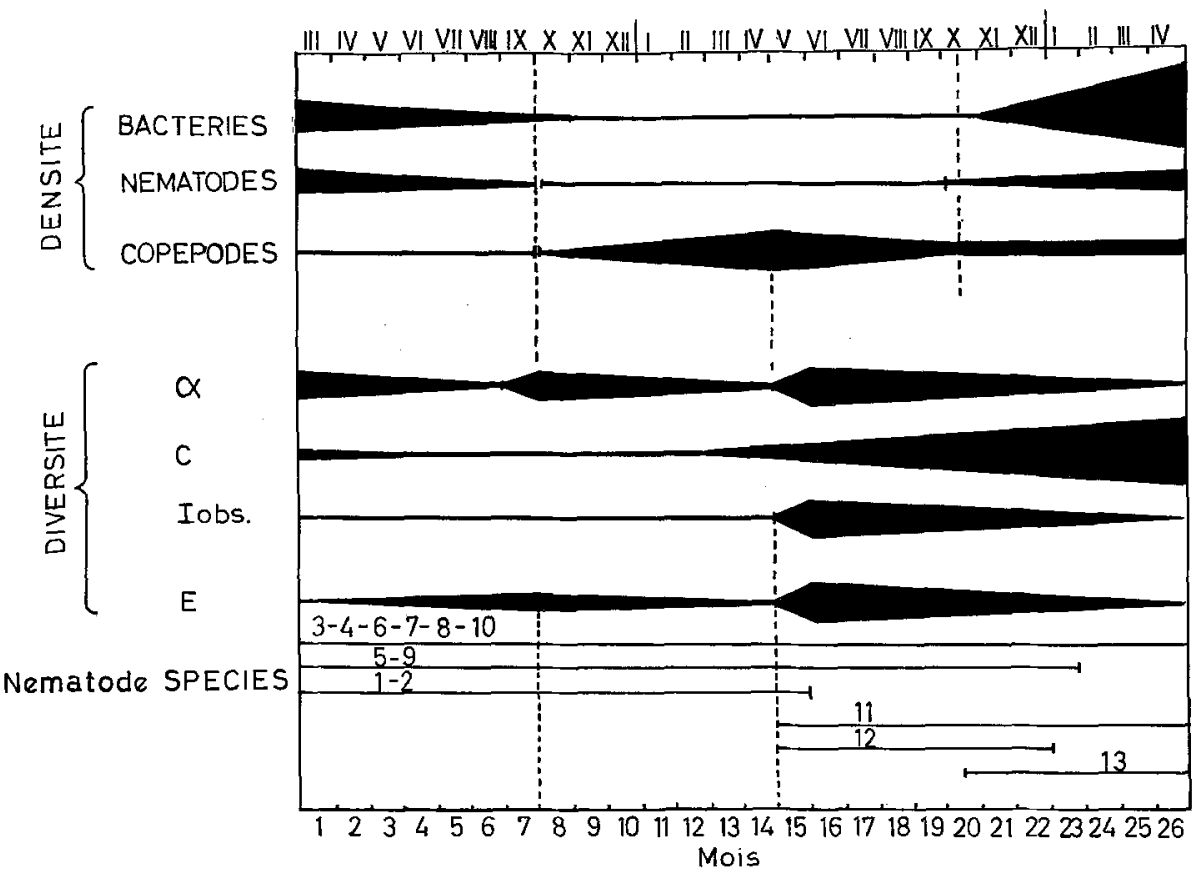

Fig. 8: Schéma d'évolution des principaux composants de l'écosystème 
Ce phénomène de concentration des Nématodes, dans le sable dragué, dément l'interprétation préliminaire que nous avions fournie pour expliquer le bloom de départ dans le système expérimental (Boucher \& Chamroux, 1976). Le point zéro utilisé pour évaluer les densités d'organismes était un carottage réalisé en plongée dans le milieu naturel. Des vérifications ultérieures nous permettent de comprendre pourquoi le sable utilisé pour remplir le bac expérimental est beaucoup plus riche en nématodes que prévu par les carottages en plongée. La très forte augmentation des densités au départ de l'expérience n'était en fait qu'un artefact.

\section{DISCUSSION}

L'évolution des Bactéries, des Copépodes et des Nématodes résumée dans la figure 8 montre l'existence de trois phases essentielles. Pendant les sept à huit premiers mois, les densités de Nématodes et de Bactéries, anormalement fortes, provoquées par le mode de prélèvement $\mathrm{d} u$ sédiment se stabilisent à un niveau comparable à celui du milieu d'origine, alors que celles des Copépodes restent faibles et constantes. Le nombre d'espèces rares de Nématodes décroît lentement, mais les relations d'abondance des espèces dominantes tendent vers un équilibre maximum proche de la nature.

Du septième au quinzième mois, les Copépodes subissent une croissance exponentielle due au développement d'une seule espèce alors que le peuplement de Nématodes reste stable quantitativement et qualitativement.

Après le quinzième mois, les densités de l'espèce dominante de Copépodes tendent vers un équilibre. La diversité des Nématodes commence à fluctuer en diminuant rapidement bien que leur densité reste constante jusqu'au dix-neuvième mois. Le développement brutal de quelques espèces, rares dans le peuplement d'origine, qui se superposent aux autres espèces en régression, produit des variations oscillatoires de la diversité significatives d'une rupture d'équilibre.

Trois faits sont à souligner:

(1) La stabilité numérique à un niveau proche de celui observé dans le milieu naturel ne constitue pas un indice suffisant de bon fonctionnement d'un écosystème, puisque la diversité des Nématodes fluctue et diminue avant que la densité ne change.

(2) La diversité élevée des Nématodes, maintenue pendant quatorze mois, reste comparable, si ce n'est supérieure, à celle des communautés naturelles (Warwick \& Buchanan, 1970; Vitiello, 1972; Heip \& Decraemer, 1974; de Bovée, 1975). Elle souligne l'intérêt de ce groupe comme test de fonctionnement de l'écosystème.

(3) Le succès d'une seule espèce épibenthique de Copépodes à partir du septième mois montre que cette espèce est en état d'équilibre dynamique avec le milieu, selon la théorie de Nicholson (1954). Ce type d'évolution correspond à l'accumulation de la nourriture pendant les sept premiers mois. Comme ce fut le cas dans l'étude de McIntyre et al. (1970), il s'avère difficile de maintenir une population multispécifique de Copépodes dans des bacs expérimentaux. Les Copépodes semblent donc un matéeiel moins pratique que les Nématodes pour l'étude de l'évolution de la structure d'un écosystème sédimentaire. Par contre, il faut souligner l'intérêt d'Ampbiascus minutus pour les études autécologiques. 
Nous pouvons nous interroger sur l'intérêt de tels microcosmes pour des études écologiques. La complexité des écosystèmes naturels et le fait qu'ils soient soumis aux variations difficilement contrôlables de l'environnement rend leur étude délicate. Des bacs expèrimentaux tels que celui que nous utilisons peuvent permette de maintenir un écosystème dans des conditions relativement bien définies pendant un temps assez long pour permettre une observation suivie des organismes.

Remerciements. Ce travail a été réalisé avec l'aide financière du CNEXO (Contrat ECOTRON no 76-5307. Nous remercions M. J. L. Douvillé et Melle L. Cras pour leur collaboration.

\section{LITTERATURE CITEE}

Boucher, G., 1975. Nématodes des sables fins infralitoraux de la Pierre Noire (Manche Occidentale). I. Desmodorida. Bull. Mus. natn. Hist. nat., Paris (Zool.) 195, 101-128.

- 1976. Nématodes des sables fins infralittoraux de la Pierre Noire (Manche Occidentale). II. Chromadorida. Bull. Mus. natn. Hist. nat., Paris (Zool.) 245, 25-61.

- \& Helléouët, M. N., 1977. Nématodes des sables fins infralittoraux de la Pierre Noire (Manche Occidentale). III. Araeolaimida et Monhysterida. Bull. Mus. natn. Hist. nat., Paris (Zool.) 297, 85-122.

- \& Chamroux, S., 1976. Bacteria and meiofauna in an experimental sand ecosystem. I. Material and preliminary results. J. exp. mar. Biol. Ecol. 24, 237-249.

Bovée, F. de, 1975. La nématofaune des vases autopolluées des Iles Kerguelen (Terres australes et antarctiques françaises). Cah. Biol. mar. 15, 711-720.

Fisher, R. A., Corbet, A. S. \& Williams, C. B., 1943. The relation between the number of species and the number of individuals in a random sample of an animal population. J. Anim. Ecol. 12, 42-58.

Hairston, N. G., Allan, G. D., Colwell, R. K., Futuyama, D. J., Howell, J., Lubin, M. D., Mathias, J. \& Vandermeer, J. H., 1968. The relationship between species diversity and stability: an experimental approach with protozoa and bacteria. Ecology 49, 1091-1101.

Heip, C. \& Decraemer, W., 1974. The diversity of nematode communites in the southern North Sea. J. mar. biol. Ass. U.K. 54, 251-255.

Lang, K., 1965. Copepoda Harpacticoidea from the Californian coast. K. svenska vetensk. Akad. Handl. 10 (2), 1-566.

Lasker, R., Wells, J. B. J. \& McIntyre, A. D., 1970. Growth, reproduction respiration and and carbon utilization of the sand-dwelling harpacticoid copepod, Asellopsis intermedia. J. mar. biol. Ass. U.K. 50, 147-160.

McIntyre, A. D., Munro, A. L. S. \& Steele, J. H., 1970. Energy flow in a sand ecosystem. In: Marine Food chains. Ed. by. J. H. Steele. Univ. Carol. Press, Berkeley, 19-31.

McNaughton, S. J., 1968. Structure and function in California grasslands. Ecology 49, 962-972.

Nicholson, A. J., 1954. An outline of the dynamics of animal populations. Austr. J. Zool. 2, 9-65.

Por, F., 1964. Les Harpacticoïdes (Copepoda Crustacea) des fonds meubles de Skagerak. Cah. Biol. mar. 5, 233-270.

Vitiello, P., 1972. Peuplements de Nématodes marins des fonds envasés de Provence occidentale. Thèse, Univ. Aix-Marseille, 190 pp.

Warwick, R. M. \& Buchanan, J. B., 1970. The meiofaune off the coast of Northumberland. I. The structure of the Nematode population. J. mar. biol. Ass. U.K. 50, 129-146.

Adresse de l'auteur premier: Dr. S. Chamroux

Place Georges Teissier

Station Biologique

F-29211 Roscoff

France 